\title{
Effects of Nonperturbative Improvement in Quenched Hadron Spectroscopy*
}

\author{
A. Cucchieri, T. Mendes and R. Petronzio ${ }^{\mathrm{a}}$ \\ ${ }^{a}$ Gruppo APE, Dipartimento di Fisica, Università di Roma "Tor Vergata" and INFN - Sezione di Roma \\ 2, Via della Ricerca Scientifica 1, 00133 Roma, ITALY
}

We discuss a comparative analysis of unimproved and nonperturbatively improved quenched hadron spectroscopy, on a set of 104 gauge configurations, at beta $=6.2$. We also present here our results for meson decay constants, including the constants $f_{D}$ and $f_{D_{s}}$ in the charm-quark region.

We report on our simulations of hadron spectroscopy using the improved action

$$
\begin{aligned}
S=S_{G} & +S_{W} \\
& +c_{S W} a^{5} \frac{i}{4} \sum_{x} \bar{\Psi}(x) \sigma_{\mu \nu} \hat{F}_{\mu \nu} \Psi(x),
\end{aligned}
$$

with $c_{S W}$ determined nonperturbatively by the Alpha collaboration. (For details see [1,2] and references thereof.)

Our runs consist of 104 quenched configurations, at $\beta=6.2$ and volume $24^{3} \times 48$. We consider 7 values of the hopping parameter $\kappa$, and all their nondegenerate flavor combinations. We run at the improved value $c_{S W}=1.61375065$ and also, for comparison, at $c_{S W}=0$. We have considered point-like sources for the inversion, and our analysis is done using single-elimination jackknife.

We are particularly interested in determining the quark-mass dependence of our observables. To this end, the usual bare quark mass $m_{q}=$ $1 / 2\left(1 / \kappa-1 / \kappa_{c}\right)$ is considered in the unimproved case, whereas for the improved case we use the improved mass, given by

$$
\widetilde{m}_{q} \equiv \frac{m^{R}}{Z_{m}}=m_{q}\left(1+b_{m} m_{q}\right)
$$

with the nonperturbative value $b_{m}=-0.62(3)$. We generally take functions of the symmetric mass average (i.e. a single variable) for our plots and chiral fits. In the case of mesons, for example,

\footnotetext{
*Talk presented by T. Mendes
}

we take

$$
\widetilde{m}_{q}\left(\kappa_{1}, \kappa_{2}\right) \equiv \frac{\widetilde{m}_{q}\left(\kappa_{1}\right)+\widetilde{m}_{q}\left(\kappa_{2}\right)}{2}
$$

where $\kappa_{1}$ and $\kappa_{2}$ correspond to two quark flavors. We find that for some observables this may not be a good approximation in the region of heavier quark masses. We mention two such cases: 1) octet (spin-1/2) baryon masses, which are not expected to be functions of a flavor-symmetric combination of the bare quark masses (nevertheless, we have shown in [1] that this effect is considerably attenuated when one employs the improved bare quark mass $\widetilde{m}_{q}$ ); and 2) pseudoscalar meson decay constants, for which we find considerable deviations from the "single-variable" curve in the charm-quark region. In this paper we discuss the latter case in some detail.

In order to determine $\kappa_{c}$ we use the unrenormalized current quark mass, defined as

$$
m_{W I} \equiv \frac{\left\langle\partial_{\mu}\left\{A_{\mu}^{(\text {bare })}+c_{A} a \partial_{\mu} P^{(\text {bare })}\right\} \mathcal{O}\right\rangle}{2\left\langle P^{(\text {bare })} \mathcal{O}\right\rangle} .
$$

We use $M_{P S}^{2} / M_{V}^{2}$ to get the strange-quark mass in lattice units $m_{s}$, and we then determine the inverse lattice spacing $a^{-1}$ by imposing $a^{-1} M_{V}\left(m_{s} / 2\right)=M_{K^{*}}$. We obtain the following results:

\begin{tabular}{ccc}
\hline & unimproved & improved \\
\hline$\kappa_{c}$ & $0.153230(15)$ & $0.135828(8)$ \\
$a^{-1}$ & $2.94(10) \mathrm{GeV}$ & $2.56(10) \mathrm{GeV}$ \\
\hline
\end{tabular}




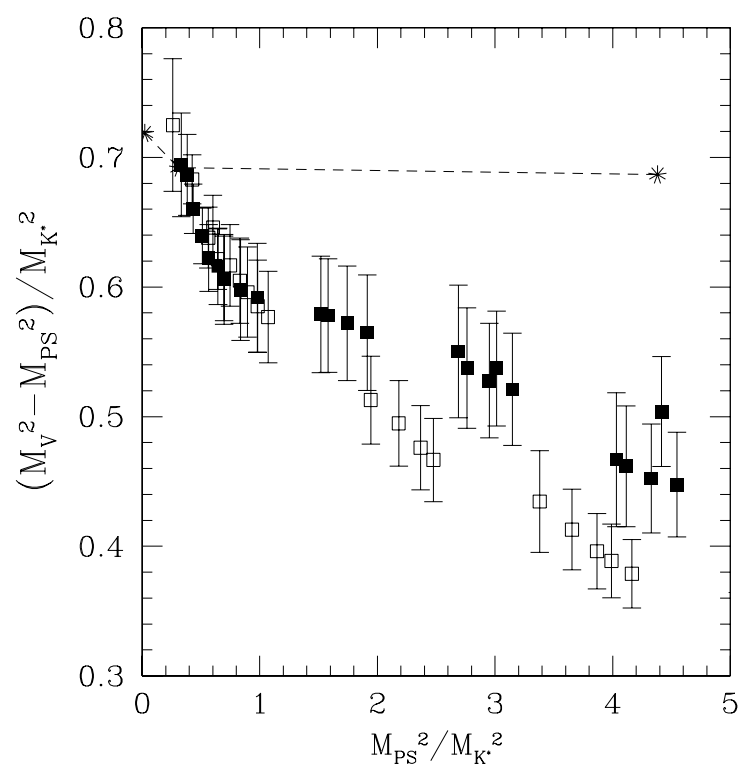

Figure 1. Hyperfine splitting in units of the $K^{*}$ meson mass. Experimental points (in the light-, strange- and charm-quark regions) are connected by a dashed line, squares and filled squares represent the unimproved and the improved case, respectively.

From our data for light-meson observables (see [1,2]) we do not see a large effect of improvement. In particular, known problems of the quenched approximation are observed also in the improved results: we see discrepancy in the value of the $J$ variable when compared with the experimental value, we get inconsistent estimates for the inverse lattice spacing depending on the quantity used as experimental input, and the hyperfine splitting between vector and pseudoscalar mesons, which is experimentally constant, drops monotonically as we move to heavier quark masses (see Fig. 1). We point out, in any case, that a slight improvement is observed in the spread of lattice spacings coming from different physical inputs (see [2]), and that, as seen in Fig. 1, although the improved and unimproved values for the hyperfine splitting are identical within error bars at lighter quark masses, the deviation from the experimental value is noticeably smaller in the improved case for heavier quark masses.

In Table 1 we present our data for the lightmeson decay constants. The difference "unim-
Table 1

Results for light-meson decay constants. The numbers in the first two rows of data are given in $\mathrm{MeV}$; their errors do not include the $4 \%$ uncertainty coming from $a^{-1}$.

\begin{tabular}{ccccc}
\hline & EXP & UNIMP & IMP & DIFF \\
\hline$f_{\pi}$ & 131 & $137(10)$ & $132(8)$ & $5(5)$ \\
$f_{K}$ & 160 & $154(7)$ & $152(6)$ & $2(3)$ \\
$f_{\pi} / M_{\rho}$ & 0.17 & $0.172(15)$ & $0.167(15)$ & $0.005(11)$ \\
$f_{K} / f_{\pi}$ & 1.22 & $1.122(36)$ & $1.149(29)$ & $-0.027(26)$ \\
$f_{\rho}^{-1}$ & 0.28 & $0.302(13)$ & $0.255(18)$ & $0.047(15)$ \\
\hline
\end{tabular}

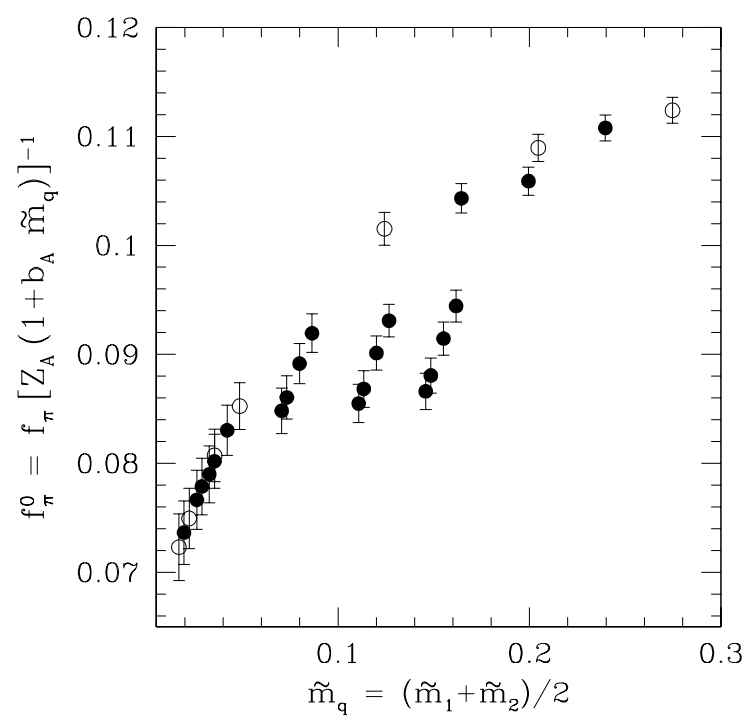

Figure 2. Pseudoscalar-meson decay constant for the improved case. Open circles correspond to diagonal flavor combinations $\left(\widetilde{m}_{1}=\widetilde{m}_{2}\right)$.

proved - improved" is computed from each jackknife cluster and subsequently averaged. We see a slight improvement in the case of $f_{\pi}$ and no improvement within error bars for the other cases. (In our evaluations for decay constants we use nonperturbative results for all the improvement coefficients and renormalization constants, except for the unimproved renormalization constant $Z_{A}$ and the improvement coefficient $b_{A}$, for which we used tadpole-improved results.)

For the case of the pseudoscalar decay constant, as seen in Fig. 2 for the improved case, the data points corresponding to nondegenerate flavor combinations (filled circles) deviate con- 
Table 2

Pseudoscalar-meson decay constants in the charm-quark region. The values in the first two rows of data are given in $\mathrm{MeV}$; their errors do not include the $4 \%$ uncertainty coming from $a^{-1}$.

\begin{tabular}{cccc}
\hline & UNIMP & IMP & DIFF \\
\hline$f_{D}$ & $208(3)$ & $201(4)$ & $7(2)$ \\
$f_{D_{s}}$ & $220(3)$ & $222(4)$ & $-2(2)$ \\
$f_{D_{s}} / f_{D}$ & $1.057(3)$ & $1.084(5)$ & $-0.027(3)$ \\
\hline
\end{tabular}

siderably from the diagonal-flavor curve, and a fit using a function of the average quark mass $\left(\widetilde{m}_{1}+\widetilde{m}_{2}\right) / 2$ is very poor. This effect is not seen in the light- and strange-quark regions, but plays a crucial role in the determination of decay constants in the charm-quark region. We have considered in this mass region a more general fit, allowing for a term of the form $\left(\widetilde{m}_{1}^{2}+\widetilde{m}_{2}^{2}\right) / 2$ in addition to the linear and quadratic terms in the average mass. This is still a symmetric function of the two quark masses $\widetilde{m}_{1}$ and $\widetilde{m}_{2}$. This fit is able to describe the numerical data with very good accuracy, and, in the light-quark region, gives results consistent with the ones of the singlevariable fit. We give our results for the constants $f_{D}$ and $f_{D_{s}}$ in Table 2. The improved data show very good agreement with the average of world lattice data given in Reference [3]. We note that a fit done using only diagonal points $\left(\widetilde{m}_{1}=\widetilde{m}_{2}\right)$ gives fit coefficients that are consistent with our more general fit. However, using the "diagonal" fit and the average-mass approximation to compute $f_{D}$ and $f_{D_{s}}$ leads to highly overestimated values: we get, in the improved case $f_{D}=265$ $\mathrm{MeV}$ and $f_{D_{s}}=275 \mathrm{MeV}$.

Finally, we have found in [1, 2] that two different determinations of the strange-quark mass are in much better agreement in the improved than in the unimproved case, and that it is very important that improved quark masses $\widetilde{m}_{q}$ be used for the determination based on the lattice ratio of charm-quark over strange-quark masses. Another sensible effect of improvement is seen in the baryon mass values, given in Table 3 in $\mathrm{MeV}$, together with their jack-knife differences. We also include here an APE plot for the decuplet baryons (Fig. 3), from which we see very clear agreement in the improved case between experimental and
Table 3

Baryon mass values in $\mathrm{MeV}$ and comparison with experiment.

\begin{tabular}{ccccc}
\hline & EXP & UNIMP & IMP & DIFF \\
\hline$M_{N}$ & 939 & $1055(97)$ & $953(117)$ & $102(117)$ \\
$M_{\Lambda}$ & 1115.7 & $1205(80)$ & $1148(76)$ & $57(74)$ \\
$M_{\Delta}$ & 1232 & $1500(150)$ & $1265(113)$ & $235(109)$ \\
$M_{\Sigma-\Lambda}$ & 73.7 & $49(23)$ & $70(29)$ & $-22(29)$ \\
$M_{\Delta-N}$ & 293 & $336(59)$ & $297(80)$ & $39(62)$ \\
\hline
\end{tabular}

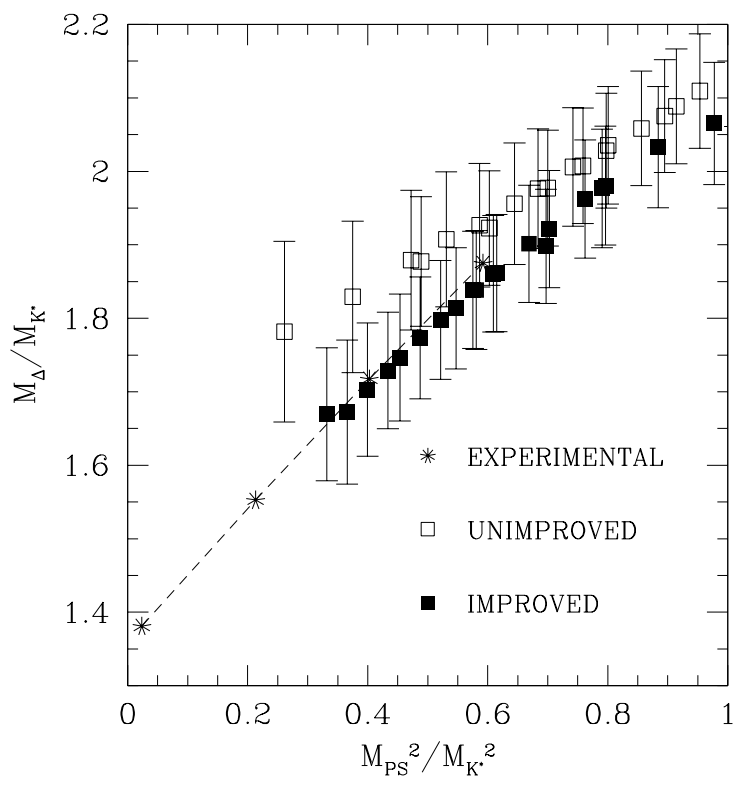

Figure 3. APE plot for decuplet baryons.

numerical points, while the unimproved points show the wrong slope. Within our error bars, we see no effect of quenching for these masses.

\section{REFERENCES}

1. A. Cucchieri, M. Masetti, T. Mendes and R. Petronzio, Phys. Lett. B422, 212 (1998);

2. A. Cucchieri, T. Mendes and R. Petronzio, J. High Energy Phys. 05, 006 (1998).

3. J.M. Flynn and C.T. Sachrajda, Heavy Quark Physics from Lattice QCD, hep-lat/9710057. 